\title{
Role of Fine Management in Maintenance of Deep Venous Indwelling Catheter in Hemodialysis Patients
}

\author{
Fei Teng \\ Zhenjiang's First People's Hospital, Zhenjiang 212002, Jiangsu Province, China
}

\begin{abstract}
[Abstract] Objective: This paper mainly explores the effect of fine management application in maintenance of deep venous indwelling catheter in hemodialysis patients. Methods: 150 hemodialysis patients with deep venous catheter indwelling in our hospital from December 2019 to September 2020 were divided into routine group and study group. The routine group was given routine nursing, and the study group was given fine management nursing. The nursing effect of the two groups was analyzed. Results: After nursing intervention, the incidence of complications in the study group was $14.66 \%$, which was lower than that in the conventional group (53.33\%), and the nursing compliance in the study group (98.66\%) was higher than that in the conventional group $(89.33 \%)$, all $\mathrm{P}<0.05$. Conclusion: Fine management plays a significant role in the maintenance of deep venous indwelling catheter in hemodialysis patients, which can reduce the occurrence of complications.
\end{abstract}

Key words: Fine management; Hemodialysis; Deep vein indwelling; Conduit maintenance; Application effect

Publication date: May, 2021; Publication online: 31 May, 2021

*Corresponding author: Fei Teng, 23936013@qq.com

\section{Introduction}

Vascular access is an indispensable condition for hemodialysis, and percutaneous deep venous catheter is a primary means to establish vascular access for hemodialysis, and it is also an important way to maintain hemodialysis, which can provide more treatment time for patients and improve their survival ${ }^{[1]}$. However, in the process of indwelling deep venous catheter, it is very easy to have complications such as catheter detachment, infection and poor blood flow, which will seriously affect the quality of life and dialysis effect of patients. Therefore, timely nursing intervention is needed to reduce the occurrence of complications and improve the dialysis effect. As a common and effective nursing method, meticulous management can make each nursing link more detailed and standardized, and provide patients with more standardized and high-quality nursing service, so as to improve the nursing effect. Based on this, this paper explores the effect of fine management in maintenance of deep venous indwelling catheter in hemodialysis patients.

\section{Material and methods}

\subsection{Basic data}

This time, 150 patients with deep venous catheter indwelling for hemodialysis in our hospital from December 2019 to September 2020 were randomly selected as the research objects. All patients met the indications of hemodialysis deep venous indwelling after clinical diagnosis, and they were informed and voluntarily signed the research consent. Exclusion criteria: Patients with severe mental illness, cognitive impairment and cancer were excluded. The proportion of male and female in the conventional group was 40: 35 , and the age range was $35-80$ years old, with an average of $(61.14 \pm 5.31)$ years old. There were 5 patients with jugular vein indwelling catheter and 70 patients with femoral vein indwelling catheter. The indwelling time of catheter was 2-180 days, with an average of $(90.34 \pm 1.36)$ days; The proportion of male and female in the study group was 39:36, with an average age of $(61.25 \pm 5.26)$ years old 
ranging from 36 to 79 years old. There were 4 patients with jugular vein indwelling catheter and 71 patients with femoral vein indwelling catheter. The indwelling time of catheter was 2-179 days, with an average indwelling time of (90.45 \pm 1.41) days. There was no significant difference in gender and age between the two groups $(\mathrm{P}>0.05)$.

\subsection{Methods}

The routine group was given routine nursing, and the nursing staff closely monitored the vital signs and catheter indwelling parts of the patients, and provided rehabilitation guidance, and timely dredged the patients' psychology ${ }^{[2]}$. The research group adopts refined management, and the specific contents are as follows:

(1) Environment management and environment play an important role in the development and control of diseases. Nursing staff need to strengthen the management of medical environment to ensure that the facilities in each ward can meet the humanized layout. Bed sheets and equipment should be placed strictly and standardized, and provide a certain range of activities for patients under the condition of ensuring safety; Open windows and ventilate regularly to make the ward air fresh, and control the indoor temperature at about $23^{\circ} \mathrm{C}$; Nurses also need to strengthen the ward cleaning care, regularly clean the floor, and combined with the actual condition of patients with comfortable position guidance, in order to eliminate the patients' strangeness and uneasiness.

(2) Before catheter indwelling care, nurses need to explain the relevant precautions to patients, and inform them of the importance and necessity of deep vein catheterization, so that patients can actively cooperate with the work; During catheter indwelling, nurses must actively cooperate with doctors in various operations, and closely observe the peripheral blood circulation of patients' limbs to avoid adverse conditions; After catheter indwelling, nurses need to make detailed records of the patient's skin outlet, catheter external interface and lumen patency, timely assess the risk of complications, and timely carry out maintenance and follow-up nursing.

(3) For health education, nurses need to give timely health knowledge education to patients and their families according to the psychological characteristics of patients, and individualized health education can be given to patients through oral education, graphic publicity or video playback, so as to ensure the effectiveness and continuity of nursing and prevent complications.Especially for the elderly patients, health education must be carried out in time to avoid the occurrence of adverse nursing risks ${ }^{[3]}$.

(4) Psychological nursing, because hemodialysis takes a long time, so patients' psychology will inevitably produce negative emotions, so psychological nursing needs to be carried out in time. First of all, nursing staff need to build a good nurse patient relationship, combined with the specific level of education and cognitive differences of patients, choose the appropriate way of communication, actively communicate with patients, master the specific psychological state of patients, and at the same time, give all kinds of care to improve patient compliance. Secondly, we need to strengthen communication with patients' family members. Good family support is of great help to patients' treatment. Therefore, we must actively encourage family members to communicate with patients more and give more care and understanding, so as to improve patients' negative emotions such as loneliness, pessimism and desolation, relieve patients' mental pressure, and patients have insufficient knowledge of hemodialysis, so it is very easy to produce adverse reactions For all kinds of psychological problems, nursing staff must actively and enthusiastically guide patients, and inform patients of dialysis environment and precautions, so as to improve patients' psychological state and prevent complications of hemodialysis.

\subsection{Observation indexes}

The incidence of complications in the two groups were statistically observed, mainly including decannulation, bleeding, embolism, catheter infection, etc; At the same time, the Frankl scale was used to evaluate the patients' treatment and nursing compliance. The score was 4 points. If the score was within 3-4 points, it was full compliance; If the score is within 2-3, it is basic compliance; if the score is within 1-2, it is non-compliance; Total compliance rate $=$ (complete compliance + basic compliance) $/$ total cases $\times 100 \%$.

\subsection{Statistical analysis}

SPSS 23.0 was used to calculate the data, and $T$ and $\chi^{2}$ tests were performed, which were expressed as $\left(\mathrm{x}^{ \pm} \mathrm{s}\right)$ and $(\mathrm{n} / \%)$. If $P<0.05$, there was a difference in the data.

\section{Results}

\subsection{The incidence of complications in the two groups was statistically analyzed.}

As shown in Table 1, the incidence of complications in the study group was significantly lower than that in the conventional group, $P<0.05$. 
Table 1. Statistics of the incidence of complications in the two groups (n /\%)

\begin{tabular}{ccccccc}
\hline Group & $\mathrm{N}$ & Get rid of control & Hemorrhage & Infection occurrence & Embolism & Total incidence \\
\hline $\begin{array}{c}\text { Routine } \\
\text { group }\end{array}$ & 75 & 7 & 14 & 10 & 9 & 53.33 \\
$\begin{array}{c}\text { Research } \\
\text { group }\end{array}$ & 75 & 1 & 5 & 3 & 2 & 14.66 \\
$\chi^{2}$ & & 4.7535 & 4.8815 & 4.1269 & 4.8071 & 24.9851 \\
P & 0.0292 & 0.0271 & 0.0422 & 0.0283 & 0.0001 \\
\hline
\end{tabular}

\subsection{Statistics of patient compliance}

was higher than that of the routine group, $P<0.05$.

As shown in Table 2, the compliance rate of the study group

Table 2. Statistics of compliance rate of the two groups (n /\%)

\begin{tabular}{cccccc}
\hline Group & $\mathrm{N}$ & Full compliance & Basic compliance & Noncompliance & Total compliance rate \\
\hline $\begin{array}{c}\text { Routine } \\
\text { group }\end{array}$ & 75 & 30 & 37 & 8 & 89.33 \\
Research & 75 & 34 & 40 & 1 & 98.66 \\
group & & & & & 5.7920 \\
$\chi^{2}$ & & & & & 0.0160 \\
P & & & & & \\
\hline
\end{tabular}

\section{Discussion}

The establishment and maintenance of hemodialysis vascular access can effectively ensure the good effect of hemodialysis treatment. Through the establishment of a good vascular access, it can provide sufficient blood flow for treatment, so as to reduce complications. Therefore, in clinical practice, nursing intervention must be taken in time to improve the maintenance effect of vascular access and improve the treatment effect of hemodialysis ${ }^{[4]}$.

Fine management is an effective nursing method commonly used in clinical practice. According to nursing procedures and methods, this method gives effective planning, organization, coordination and control to the operation process of nursing procedures, so as to effectively improve the quality of nursing. Through the detailed and standardized treatment of each routine nursing process, the nursing links can be effectively optimized, which can provide reference for clinical practice Patients provide a more professional and standardized nursing intervention to improve the nursing effect ${ }^{[5-9]}$. Through health education and psychological nursing, we can effectively improve the psychological state of patients, and ensure that patients master basic hemodialysis knowledge, understand the importance of deep vein catheterization in hemodialysis, improve compliance, at the same time, strengthen various environmental nursing, provide patients with a comfortable and quiet nursing environment, and improve the nursing effect; Finally, actively communicate with patients' family members, strengthen patient communication, enhance nurse patient relationship, to ensure that patients' family members can cooperate with nursing staff to participate in nursing work, so as to better improve the clinical situation of patients and improve the effect of hemodialysis.

In conclusion, the application of fine management in hemodialysis patients with deep vein indwelling catheter maintenance effect is very significant, can reduce the occurrence of complications.

\section{References}

[1] An HD, Qi XM, Wang R, et al. The effect of Gracz internal arteriovenous fistula and deep venous catheter indwelling on the prognosis of patients with hemodialysis $[\mathrm{J}]$. Chinese Blood Purification, 2020, 19(7): 471-475.

[2] Chen RL. Effect of evidence-based nursing intervention on complications related to deep vein indwelling 
catheter in patients with uremia hemodialysis [J]. Dialysis and Artificial Organs, 2019, 30(4): 157-159.

[3] Du BP, Xiao SM. Effects of fine management measures on the incidence of catheter detachment and catheter-related infection in deep vein catheters on hemodialysis [J]. Modern Diagnosis and Treatment, 2020, 31(316(16)): 135-136.

[4] Liu L. Analysis of clinical nursing path in hemodialysis patients with temporary indwelling deep vein double-lumen dialysis catheter $[\mathrm{J}]$. Journal of Cardiovascular Surgery (Electronic Edition), 2019, 8(2): 181-182.

[5] Zheng YT. Effect of evidence-based nursing on vascular access maintenance compliance and prevention of adverse conditions in patients with indwelling hemodialysis catheter $[\mathrm{J}]$. International Journal of Nursing, 2020, 39(16): 2910-2913.

[6] Li XY, Lei S, Liu ZJ. Comparison of long-term indwelling catheter of deep vein and internal arteriovenous fistula in hemodialysis patients $[\mathrm{J}]$. World Latest Medical Information Abstracts, 2019, 19(29): $65 \& 69$.

[7] Liu XL, Li GY, Wang XY, et al. The effect of early urokinase on the formation of fibrin sheath in chronic renal failure patients with intravenous catheterization [J]. Clinical Misdiagnosis and Mistreatment, 2020, 33(4): 52-56.

[8] Zhou X, Yao Y, Zhou XL, et al. A meta-analysis of the efficacy of central intravenous catheterization with antibiotics and heparin in preventing catheterization associated infections in hemodialysis patients [J]. Journal of Clinical Nephrology, 2019(8).

[9] Ye WH, Gao Q, Liu J. The effect of multiple education models on the compliance and adverse psychological status of patients with long-term indwenting double-lumen catheter maintenance in hemodialysis $[\mathrm{J}]$ Chinese Journal of Blood Purification, 2020, 19(2): 67-70. 